J. MED. MICROBIOL.--VOL. 16 (1983) 467-475

(C) 1983 The Pathological Society of Great Britain and Ireland

\title{
ASPECTS OF THE PATHOGENICITY OF SOME ORAL AND OTHER HAEMOPHILI
}

\author{
J. E. TuYau AND W. Sims
}

Department of Microbiology and Preventive Dentistry, Royal Dental Hospital, Leicester
Square, London, WC2H $7 \mathrm{LJ}$

SUmmaRY. The pathogenicity of the predominantly non-capsulated, $\mathrm{V}$-factor requiring haemophili that are commonly recovered from oral infections has been explored by studies of their endotoxins and infectivity as compared with those of Haemophilus influenzae. Similar yields of endotoxin $(2 \cdot 40-2.59 \% \mathrm{w} / \mathrm{w})$ were obtained from all the haemophili examined except $H$. haemolyticus $(1.61 \% \mathrm{w} / \mathrm{w})$. The lipopolysaccharide (LPS) extracts all contained heptoses but not 2-keto-3-deoxyoctonate (KDO). The fatty acid compositions of the lipid-A fractions were essentially similar but comprised $76 \%$ of the LPS in the $H$. influenzae type d strain tested and only $20 \%$ in the $H$. influenzae type b strain and some strains of $H$. parainfluenzae. All extracts contained arachidic acid, which may be unique to haemophili. The endotoxins from all strains produced characteristic pyrogenic and polymorph effects in rabbits. The endotoxins from the pharyngeal Xand V-factor-requiring strains had LD50 values for actinomycin-Dsensitised mice of $2.4-2.7 \mu \mathrm{g} / \mathrm{kg}$, and were about eight times more potent than those from the oral V-factor-requiring strains (LD50 values $17 \cdot 2-22 \cdot 4 \mu \mathrm{g} / \mathrm{ml}$ ). Approximately ten thousand times more free endotoxin was detected in broth cultures of $H$. influenzae type b than in those of oral haemophili, and this greater endotoxin release was not associated with a greater degree of autolysis. Endotoxin release from viable cells may be important in the pathogenicity of this organism. $H$. influenzae type $\mathrm{b}$ was much more potent in producing infection in chambers implanted subcutaneously in guinea pigs than were oral strains of haemophili; only 10 type b organisms were required, compared with $9 \times 10^{5}$ of $H$. parainfluenzae. However, in infections maintained for 90 days, the numbers of haemophili-c. $10^{7} / \mathrm{ml}$ of chamber fluid-were similar for all the test strains. Thus, although the oral haemophili lack special attributes of invasiveness and resistance to host defences, they are not devoid of pathogenic potential and, if allowed to proliferate, may become an important element in an infection. 


\section{INTRODUCTION}

Although viridans streptococci are the bacteria consistently found in greatest numbers in material from purulent oral infections, haemophili are also commonly present and are occasionally the predominant organisms (Sims, 1974). Most of the haemophili recovered require $\mathrm{V}$ factor (nicotinamide adenine dinucleotide) but not $\mathrm{X}$ factor (haematin) for growth; non-capsulated strains of Haemophilus influenzae (requiring both factors) are also isolated, though less frequently. Approximately $15 \%$ of oral Haemophilus strains produce $\beta$-haemolysis on horse blood agar. There is no clear way of assessing the contribution that these comparatively non-pathogenic species of haemophili, which are part of the normal oral flora (Sims, 1970), make to the progress of oral infections. However, we have examined the endotoxins and some other aspects of the pathogenicity of these strains, and have compared them with those of capsulated strains of $H$. influenzae, including one strain belonging to type $b$, the serotype responsible for the great majority of serious haemophilus infections.

\section{MATERIALS AND METHODS}

Bacteria. The strains examined were: Haemophilus influenzae, capsulated strains types a-d, NCTC nos. 8465, 7279, 8469 and 8470; an X-factor dependent Haemophilus strain NCTC 10555; one strain of $H$. haemolyticus; two strains of $H$. influenzae (non-capsulated); and six strains each of $H$. parainfluenzae and $H$. parahaemolyticus, isolated from purulent infections of the oral cavity.

Preparation of cell walls. Haemophili were grown on brain-heart-infusion heated-blood agar for $18 \mathrm{~h}$ at $37^{\circ} \mathrm{C}$. They were harvested in distilled water, washed, resuspended to a concentration of approx. $8 \%$ and disrupted by sonic disintegration for 2 min. After centrifugation for $30 \mathrm{~min}$ at $10000 \mathrm{~g}$ and $4^{\circ} \mathrm{C}$, the pellet was freeze dried and reconstituted in endotoxin-free distilled water to a concentration of $1 \mathrm{mg} / \mathrm{ml}$.

Limulus amoebocyte lysate ( $L A L$ ) assays were performed according to Technical Bulletin No. 210, 1973, Sigma Chemical Co., Poole, Dorset.

Extraction of endotoxin. A modification of the phenol-water method of Westphal and Jann (1965) was employed. The haemophili were grown on plates of brain-heart-infusion heated-blood agar for $24 \mathrm{~h}$ at $37^{\circ} \mathrm{C}$ and harvested in distilled water. They were washed once and resuspended at a concentration of $200 \mathrm{mg} / \mathrm{ml}$ in a flask with glass beads. An equal volume of $90 \%$ phenol-water was added and the flask was heated at $68^{\circ} \mathrm{C}$ for 15 min with regular mixing, and then immersed in an ice-bath. After centrifugation, the aqueous layer was collected, the residue taken up in an equal volume of distilled water and the procedure repeated. The pooled aqueous layers were dialysed overnight against running tap water and then centrifuged at 10000 $g$ for $30 \mathrm{~min}$ at $4^{\circ} \mathrm{C}$ to remove insoluble matter. The supernate was freeze-dried, reconstituted to $2 \% \mathrm{w} / \mathrm{v}$ in distilled water and centrifuged at $10000 \mathrm{~g}$ for $2 \mathrm{~h}$ at $4^{\circ} \mathrm{C}$. The gelatinous pellet was mixed in an equal volume of distilled water and centrifuged again. The residue was taken up in a little distilled water and freeze-dried.

Preparation, hydrolysis and methylation of lipid $A$. Endotoxin was dissolved to a concentration of $0.2 \%$ in aqueous acetic acid $1.0 \% \mathrm{w} / \mathrm{v}$ and heated at $100{ }^{\circ} \mathrm{C}$ for $2 \mathrm{~h}$. The precipitate was washed and then hydrolysed in $4 \mathrm{~N} \mathrm{HCl}$ in a sealed ampoule for $5 \mathrm{~h}$ at $100^{\circ} \mathrm{C}$; the $\mathrm{HCl}$ was removed by evaporation in a vacuum dessicator over phosphorus pentoxide. Weighed amounts of this hydrolysed lipid A were dissolved in 1-ml amounts of distilled water. The internal standard $\mathrm{n}$-heptadecanoic acid was added, followed by $0.4 \mathrm{ml}$ of $\mathrm{H}_{2} \mathrm{SO}_{4} 50 \%$ and $2.0 \mathrm{ml}$ of methanol, and the mixture was incubated at $56^{\circ} \mathrm{C}$ for $30 \mathrm{~min}$. A further $1.0 \mathrm{ml}$ of water was added and mixed, followed by $0.5 \mathrm{ml}$ of chloroform. The tube was inverted 20 times and lightly centrifuged. One $\mu \mathrm{l}$ of the chloroform layer was analysed by gas chromatography.

Gas chromatography. Lipids were separated in a glass column containing diethylene glycol succinate $20 \%$ on chromosorb W $\left(80-120\right.$ mesh) at $156^{\circ} \mathrm{C}$. The carrier gas was nitrogen at a flow 
rate of $30 \mathrm{ml} / \mathrm{min}$, and detection was by flame ionization with a hydrogen and air combustion mixture.

Estimation of heptoses and 2-keto-3-deoxyoctonic acid (KDO). The methods of Osborn (1963) were used.

Estimation of autolysis and free endotoxin. Haemophili were grown in brain-heart-infusion broth supplemented with haematin and NAD $10 \mu \mathrm{g} / \mathrm{ml}$ for $18 \mathrm{~h}$ at $37^{\circ} \mathrm{C}$. The culture was divided into two portions; one was centrifuged at $10000 \mathrm{~g}$ for $30 \mathrm{~min}$. The method of Meynell and Meynell (1965) was used to measure the DNA in the uncentrifuged broth and in the supernate of the other portion, and the endotoxin content of the latter was estimated by LAL assay. A portion of the supernate was centrifuged at $10000 \mathrm{~g}$ for a further $2 \mathrm{~h}$ at $4^{\circ} \mathrm{C}$. The pellet was redissolved in a little distilled water and mixed with an equal volume of phosphotungstic acid $(p \mathrm{H} \mathrm{7.2)} \mathrm{3 \%}$, and drops of this solution were applied to 400 mesh carbon-formovar coated grids for electron microscopy.

Animal experiments. Perforated polyallomer tubes were implanted subcutaneously in guinea pigs, by the technique of Veale et al. (1975). The chambers were left for 30-60 days to encapsulate and fill with exudate. Ten-fold dilutions of haemophilus suspensions in phosphate buffered saline were injected into the chambers, and $0 \cdot 2 \mathrm{ml}$ samples were withdrawn $7,15,30,45$, 60 and 90 days later. The samples and serial dilutions thereof were spread on plates of heated blood agar and incubated at $37^{\circ} \mathrm{C}$ in air plus $5 \% \mathrm{CO}_{2}$.

Seven groups of six mice each were given intraperitoneal injections of $12.5 \mu \mathrm{g}$ of actinomycin $\mathrm{D} /$ mouse; $3 \mathrm{~h}$ later, six groups were given injections of graded amounts of endotoxin by the same route. Deaths were recorded during a period of 7 days. The LD50 was estimated by the method of Reed and Muench (1938).

Endotoxin in various amounts $(1-500 \mu \mathrm{g})$ was injected intravenously into rabbits; the rectal temperature was recorded and blood sampled after 5, 15,30 and $60 \mathrm{~min}$ and hourly thereafter for $6 \mathrm{~h}$. Fatty acids either dissolved in ethanol or complexed with bovine albumin at a concentration of $0.3 \mathrm{mmol}$ were injected intravenously in $0.5-\mathrm{ml}$ amounts into other rabbits, and the response was monitored as before.

\section{RESULTS}

\section{Yield and composition of extracted endotoxin}

A standard LAL preparation was gelled by 1 in 1000 dilutions of suspensions of the lyophilised cell walls of each of the strains of Haemophilus studied. Extracted LPS of any strain at a concentration of $1.0 \mu \mathrm{g} / \mathrm{ml}$ also gelled the LAL.

The yield of extracted LPS from whole cells was $2 \cdot 40-2 \cdot 59 \%(w / w)$, except with the oral strain of $H$. haemolyticus from which only $1.61 \%$ was obtained. Examination of the polysaccharide fraction of each of the LPS preparations showed absorption maxima at wavelengths of 400 and $505 \mathrm{~nm}$, which indicates the presence of 6-deoxyheptoses and heptoses; 2-keto-3-deoxyoctonate (KDO) was not detected in any extract.

Examination of lipid-A portions of the LPS showed that all strains of Haemophilus contained a C20:0 fatty acid, arachidic acid, which has not been reported previously in the LPS of other bacteria. The lipid A of most strains contained myristic, palmitic, stearic, oleic and linoleic acids, and that of some strains contained trace amounts of lauric and palmitoleic acids. Two representative gas chromatograms of the fatty acids obtained by hydrolysis and methylation of lipid A are shown in figs. 1 and 2. The $H$. influenzae type $\mathrm{b}$ strain differed from all the other strains tested in that only myristic, stearic and arachidic acids were detected in the lipid-A moiety, and they comprised only $19 \%$ of the LPS. In contrast, the $H$. influenzae type d strain contained the full 


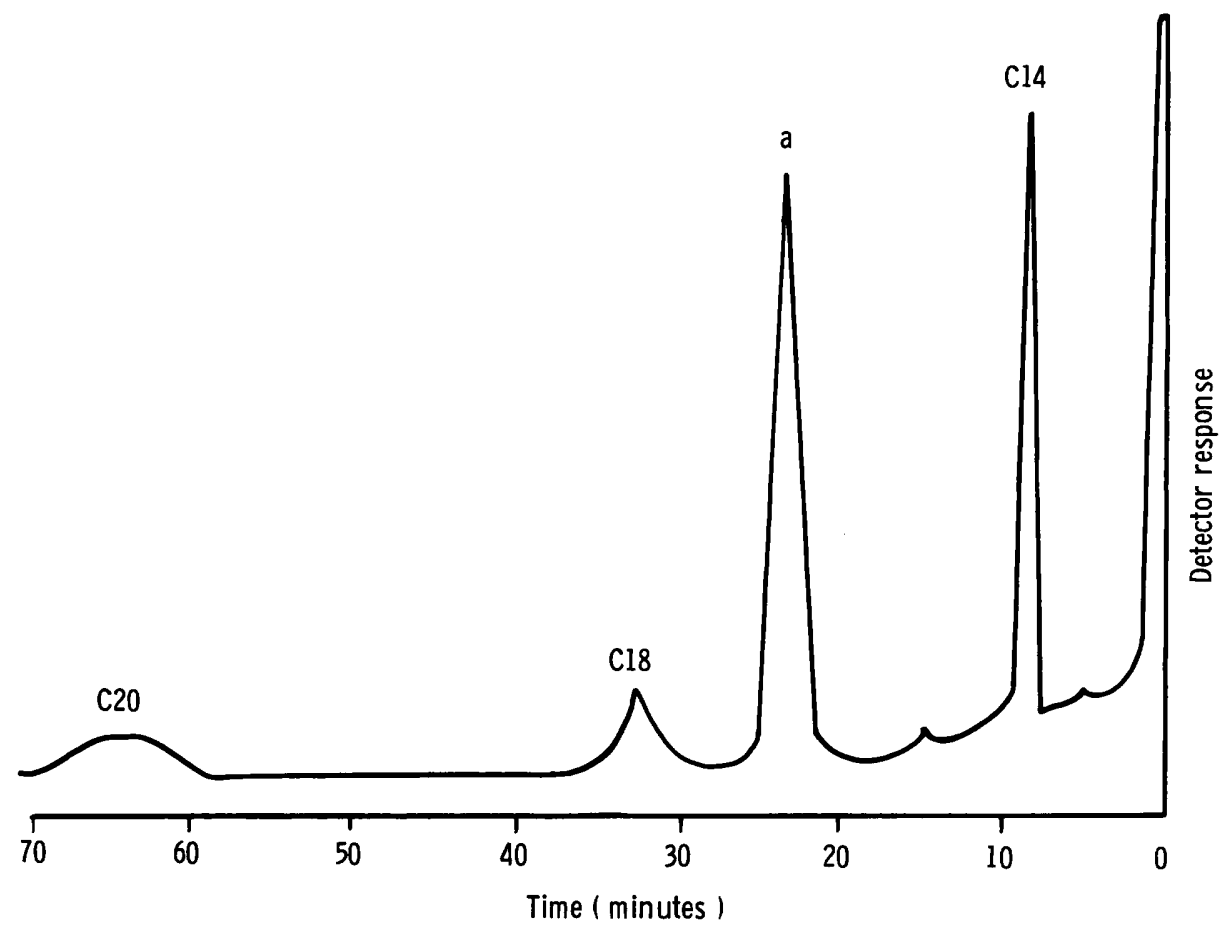

FIG. 1.-Gas chromatograph recording of the fatty acids separated from the hydrolysed, methylated lipid-A of $H$. influenzae type b, NCTC8465. C14 is myristic acid, C18 stearic acid and C20 arichidic acid. $\mathrm{a}=$ internal standard C17 n-heptadecanoic (margaric) acid.

range of fatty acids, and they amounted to $76 \%$ of the LPS. $H$. influenzae types a and c, like type $b$, were devoid of lauric acid. Some strains of $H$. parainfluenzae, although containing all the fatty acids mentioned, resembled $H$. influenzae type $\mathrm{b}$ in that the fatty acids constituted only $20 \%$ of the LPS.

\section{Effects of extracted endotoxins and fatty acids}

The LD50 values of some extracted endotoxins for actinomycin-D-sensitised mice are listed in table I. The endotoxins from capsulated and non-capsulated $H$. influenzae were of comparable potency and some six to eight times more lethal than those extracted from $H$. parahaemolyticus and $H$. parainfluenzae.

When extracted endotoxin was injected intravenously into rabbits the characteristic changes in blood counts of polymorphs (fig. 3) and lymphocytes and in body temperature were observed. No differences in potency were demonstrable with endotoxins obtained from different species of Haemophilus. The individual lipid-A fatty acids, as well as $\mathrm{C} 22$ behenic acid included for comparison, all produced the changes in leukocyte counts but no evidence of pyrogenicity. When these acids were dissolved in bovine albumin instead of ethanol the changes in body temperature did occur. The effects of arachidic acid, which appears to be peculiar to haemophilus endotoxin, were indistinguishable from those of the other fatty acids found in endotoxins. 


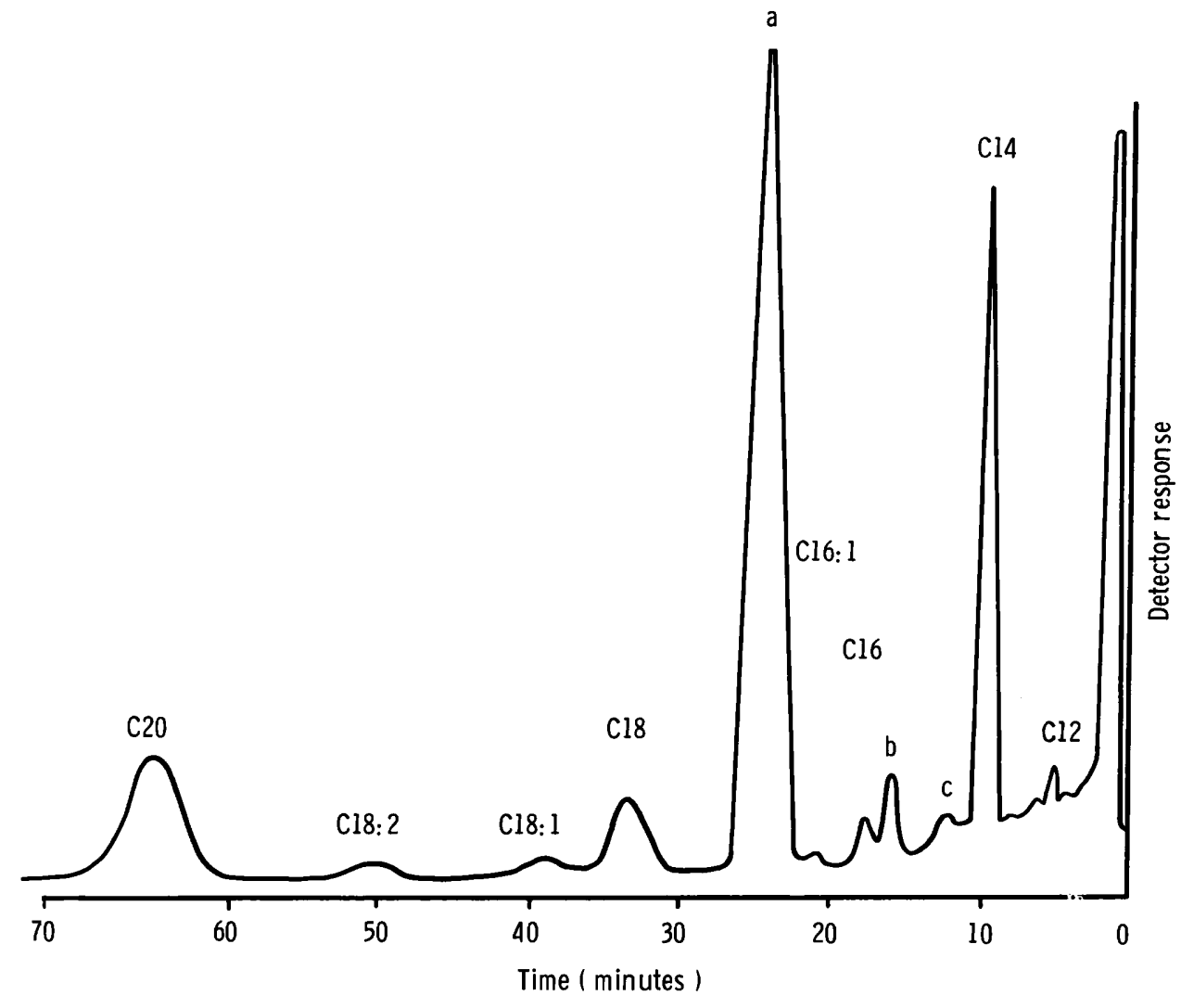

FiG. 2.-Gas chromatograph recording of the fatty acids separated from the hydrolysed, methylated lipid A of an oral strain of H. parahaemolyticus. C12 is lauric acid, C14 myristic acid, C16 palmitic acid, C16:1 palmitoleic acid, $\mathrm{C} 18$ stearic acid, C18:1 oleic acid, C18:2 linoleic acid and C20 arichidic acid; $\mathrm{a}=$ internal standard $\mathrm{Cl} 7 \mathrm{n}$-heptadecanoic (margaric) acid; $\mathrm{b}$ and $\mathrm{c}$ were presumed to be breakdown products of hydroxy-myristic acid.

TABLE I

LD50 of haemophilus endotoxin for mice sensitised with actinomycin D

\begin{tabular}{lr}
\hline Organism & $\mathrm{LD} 50(\mu \mathrm{g} / \mathrm{kg})$ \\
\hline H. influenzae type b, NCTC7279 & $2 \cdot 41$ \\
H. influenzae, non-capsulated, oral strain & $2 \cdot 69$ \\
H. parahaemolyticus, oral strain & $17 \cdot 20$ \\
H. parainfluenzae, oral strain & $22 \cdot 44$ \\
\hline
\end{tabular}

In tables I-III the results shown, other than for $H$. influenzae type b, are representative-more than one strain was tested in each case.

\section{Release of endotoxin}

The amount of endotoxin found in the supernates of 18-h broth cultures of haemophili varied markedly among species and was not proportional to the degree of cell lysis estimated by DNA release (table II). $H$. influenzae type b and 


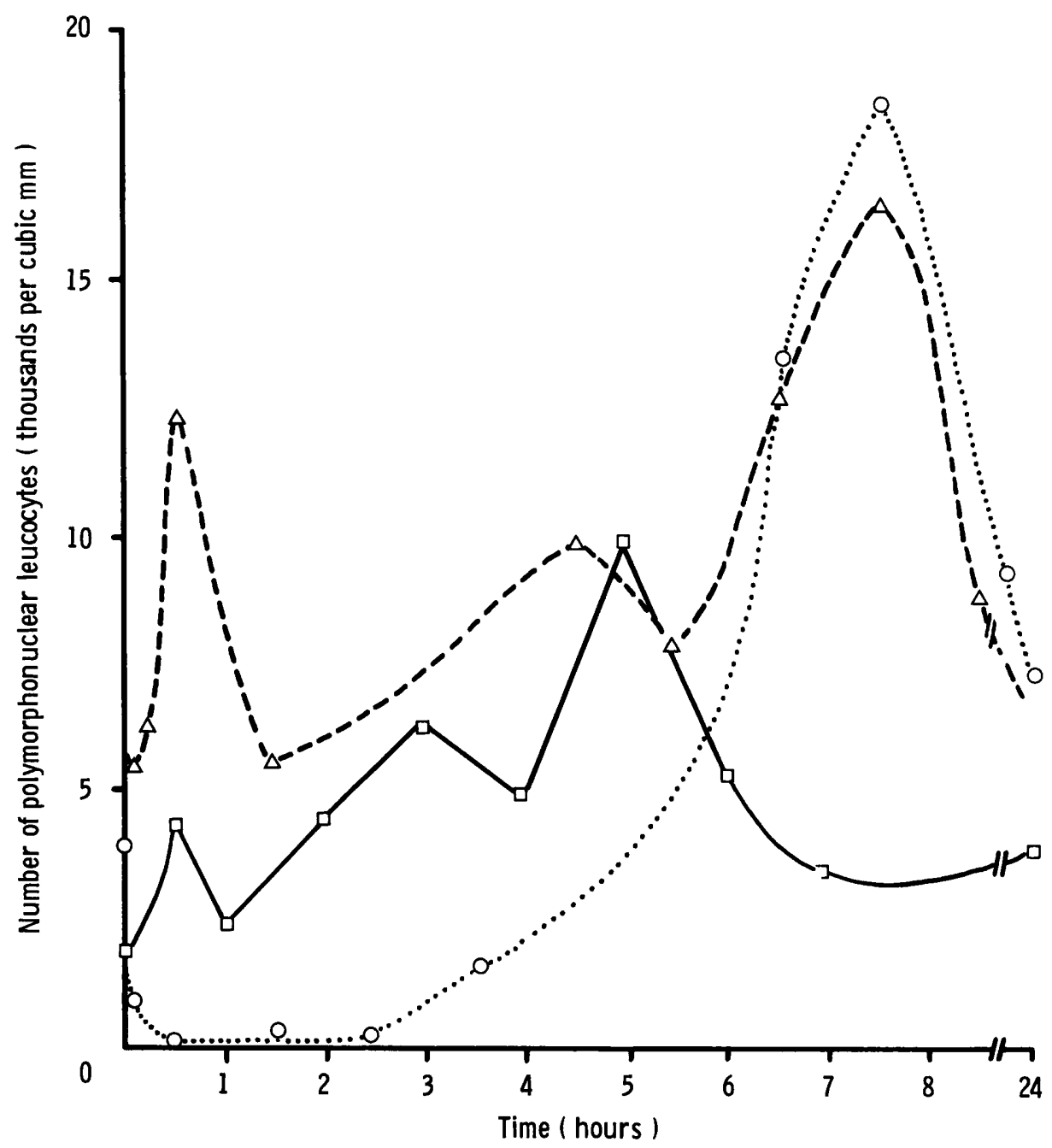

FIG. 3.-Changes in numbers of circulating polmorphs in rabbits after the intravenous injection of $100 \mu \mathrm{g}$ of endotoxin extracted from $H$. influenzae type b, NCTC7279 $(\Delta--\Delta), 100 \mu \mathrm{g}$ of endotoxin from an oral strain of $H$. parahaemolyticus $(0 \cdots O)$, and $0.5 \mathrm{ml}$ of a $0.3 \mathrm{mM}$ solution of arachidic acid complexed with bovine albumin $(\square-\square)$.

TABLE II

LAL titres of haemophilus broth supernates compared with the degrees of cell lysis estimated by measuring released DNA

\begin{tabular}{lcc}
\hline Organism & $\begin{array}{c}\text { Highest broth dilution } \\
\text { that caused LAL to gel }\end{array}$ & $\begin{array}{c}\text { Percentage } \\
\text { autolysis }\end{array}$ \\
\hline H. influenzae type b, NCTC7279 & $10^{9}$ & $39 \cdot 4$ \\
H. influenzae, non-capsulated, oral strain & $10^{7}$ & $23 \cdot 0$ \\
H. parahaemolyticus, oral strain & $10^{5}$ & $37 \cdot 3$ \\
H. parainfluenzae, oral strain & $10^{5}$ & $47 \cdot 9$ \\
Uninoculated broth (control) & $10^{3}$ & $\ldots$ \\
\hline
\end{tabular}


$H$. parahaemolyticus underwent similar degrees of autolysis but the $H$. influenzae type b strain released $10^{4}$ times more endotoxin. The amount of endotoxin that could be extracted from these two species was similar $(2.59 \%$ and $2.49 \% \mathrm{w} / \mathrm{w}$ respectively); therefore, it appears that non-autolysing cells of $H$. influenzae release endotoxin. In support of this hypothesis, free blebs of endotoxin were demonstrated by electron microscopy in the deposits from ultracentrifuged broth supernates of $H$. influenzae type $b$, but not of the other strains of haemophili.

\section{Infections of subcutaneously implanted chambers}

The numbers of haemophili required to establish infections that would persist for at least 90 days in chambers implanted subcutaneously in guinea-pigs are shown in table III. The $H$. influenzae type b strain was much more potent than the other strains tested; only 10 organisms were required to establish infection, whereas $10^{5}-10^{6}$ organisms were required with the other strains. In infected chambers, the number of haemophili (of any strain) increased to approximately $10^{7} / \mathrm{ml}$ of exudate and remained at this level.

\section{TABLE III}

Minimum numbers of haemophili required to establish persistent infections in chambers implanted subcutaneously in guinea pigs

\begin{tabular}{lc}
\hline Organism & Number (cfu) \\
\hline H. influenzae type b, NCTC7279 & $<10$ \\
H. influenzae, non-capsulated, oral strain & $10^{5}$ \\
H. parahaemolyticus, oral strain & $3 \cdot 6 \times 10^{5}$ \\
$H$. parainfluenzae, oral strain & $9 \times 10^{5}$ \\
\hline
\end{tabular}

Attempts to use this system to study the effects of mixtures of haemophili with other organisms isolated from oral infections were unsuccessful. For example, none of the oral streptococci tested was alone able to produce persistent infections of the chambers; and when mixed with infective doses of haemophili the streptococci prevented these organisms from establishing infections - except that small inocula of $H$. influenzae type b produced infections of the chambers regardless of the admixture of large numbers of oral streptococci.

\section{Discussion}

The results of these investigations show that oral haemophili, most of which do not require haematin for growth (Sims, 1970; Kilian, 1976), are much less pathogenic than some haematin-requiring pharyngeal strains. Nevertheless, although $H$. parainfluenzae seems innocuous in comparison with $H$. influenzae type b, the oral haemophili are not altogether devoid of pathogenic potential and there are many reports of serious infections caused by them (e.g., Parsons and Faris, 1973; Chow et al., 1974; Holt et al., 1974).

The possession of a type b capsule is well known to be of crucial importance in the 
pathogenicity of haemophili, but it is clear that other factors contribute to the greater virulence of this serotype. We reported previously that most strains of haemophili produce neuraminidase (Tuyau and Sims, 1974) and, typically, $H$. influenzae type b produced this enzyme in greatest abundance. Although the yield of endotoxin from all strains was similar and such chemical comparisons as we have attempted revealed no particular differences, the endotoxins from the haematin-requiring strains were some eight times more lethal for actinomycin-D-sensitised mice. Haemophilus endotoxin is of the same order of toxicity as those obtained from other genera, but differences in toxicity of endotoxins in general await rational explanation. The differences in amount and type of fatty acid in the toxic lipid-A moiety of the haemophilus endotoxins seem unrelated to biological effect. Arachidic acid had no properties to distinguish it from the other lipid-A fatty acids and its presence is probably irrelevent in regard to pathogenicity. However, if it proves to be unique to haemophilus endotoxin, as we aver, it could be a useful taxonomic marker. Although Flesher and Insel (1978) reported traces of KDO in haemophilus endotoxin, we believe that this compound is absent and that, in this respect, haemophilus endotoxins resemble those of anaerobic gram-negative bacilli (Hofstad, 1974). We have not determined how the polysaccharide and lipid-A portions of haemophilus endotoxin are linked, but the presence of heptoses was established.

$H$. influenzae type b was included in this work for comparison, not as a principal interest, but the chance finding that it releases endotoxin without autolysis could be relevant to its pathogenicity. Free endotoxin damages macrophages and triggers the complement cascade (Morrison and Ulevitch, 1978; Bradley, 1979). Activating complement before it reaches the bacterial cell ought to favour survival of the organism, and inflammatory changes provoked by the reaction of free endotoxin and complement might facilitate invasion of mucous membranes.

The greater ability of a capsulated (type b) organism to initiate an infection was clearly shown in the subcutaneous chamber experiments in guinea pigs. Once an infection was established, however, similar numbers of capsulated or non-capsulated organisms maintained the infection. This provides an approximate parallel with the clinical situation, where it is type $\mathrm{b} H$. influenzae strains that most readily infect by invading mucous membranes and resisting host defences, but where, on the relatively few occasions when non-capsulated haemophili establish infection, it may be equally dangerous and difficult to resolve. Because of their requirement for growth factors, haemophili tend to be regarded as difficult, or slow, to grow. However, when the necessary nutrients are provided and the atmosphere is supplemented with extra carbon dioxide, haemophili grow quickly and luxuriantly. When polymorphs migrate into the mouth they remain viable only whilst protected by the surface film of mucus, and autolyse readily in surface oral wounds (Klinkhamer, 1963; Rovelstad, 1964). The consequent release of NAD could turn the growth of the haemophili of the normal oral flora, inevitably associated with such lesions, into a major cause of aggravation.

\section{REFERENCES}

BRADLEY, S. G. 1979. Cellular and molecular mechanisms of action of bacterial endotoxins. Annual Review of Microbiology, 33, 67-94.

Chow, A. W., Bushkell, L. L., Yoshikawa, T. T. ANd Guze, L. B. 1974. Haemophilus 
parainfluenzae epiglottitis with meningitis and bacteremia in an adult. The American Journal of Medical Sciences, 267, 365-368.

FLESHER, A. R. AND INSEL, R. A. 1978. Characterization of lipopolysaccharide of Haemophilus influenzae. The Journal of Infectious Diseases, 138, 719-730.

HoFsTAD, T. 1974. The distribution of heptose and 2-keto-3-deoxy-octonate in Bacteriodaceae. Journal of General Microbiology, 85, 314-320.

Holt, R. N., Taylor, C. D. Schneider, H. J. and Hallock, J. A. 1974. Three cases of Haemophilus parainfluenzae meningitis. Clinical Paediatrics, 13, 666-668.

Kilian, M. 1976. A taxonomic study of the genus Haemophilus, with the proposal of a new species. Journal of General Microbiology, 93, 9-62.

Klinkhamer, J. M. 1963. Human oral leucocytes. Periodontics, 1, 109-117.

Meynell, G. G. AND Meynell, E. 1965. Theory and practice in experimental bacteriology. Cambridge University Press, Cambridge, pp. 5-6.

Morrison, D. C. AND Ulevitch, R. J. 1978. The effects of bacterial endotoxins on host mediation systems. American Journal of Pathology, 93, 527-617.

OsBoRN, M. J. 1963. Studies on the Gram-negative cell wall. I: Evidence for the role 2-keto-3-deoxyoctonate in the lipopolysaccharide of Salmonella typhimurium. Proceedings of the National Academy of Sciences USA, 50, 499-506.

Parsons, M. AND Faris, I. 1973. Empyema of the gall bladder due to Haemophilus parahaemolyticus, with a brief review of its role as a pathogen. Journal of Clinical Pathology, 26, 604-605.

REED, L. J. AND MuENCH, H. 1983. A simple method of estimating fifty per cent end-points. American Journal of Hygiene, 27, 493-497.

Rovelstad, G. H. 1964. Salivary corpuscle activity. Journal of the American Dental Association, 68, 364-373.

Sims, W. 1970. Oral haemophili. Journal of Medical Microbiology, 3, 615-625.

SIMS, W. 1974. The clinical bacteriology of purulent oral infections. British Journal of Oral Surgery, 12, 1-12.

TUYAU, J. E. AND SIMS, W. 1974. Neuraminidase activity in human oral strains of haemophili. Archives of Oral Biology, 19, 817-819.

Veale, D. R., Smith, H., Witt, K. A. and Marshall, R. B. 1975. Differential ability of colonial types of Neisseria gonorrhoeae to produce infection and an inflammatory response in subcutaneous perforated plastic chambers in guinea-pigs and rabbits. Journal of Medical Microbiology, 8, 325-335.

WeSTPHAL, O. AND JANN, K. 1965. Bacterial lipopolysaccharides. Extraction with phenolwater and further applications of the procedure. In Methods in carbohydrate chemistry, vol. 5, edited by R. L. Whistler, Academic Press, New York, pp. 83-91. 